\title{
Effect of deposition time on the characteristics of nanocrystalline cds thin films and photodetection properties
}

\begin{abstract}
Nanocrystalline CdS thin films were grown on silicon substrates using microwave-assisted chemical bath deposition. Aqueous solutions of cadmium chloride $(\mathrm{CdCl} 2)$ and thiourea [SC(NH2)2], which served as cadmium $\mathrm{Cd} 2+$ and sulfur $\mathrm{S} 2-$ ions sources, respectively were used to synthesize the nanocrystalline CdS thin films. Morphological, structural, and optical analyses revealed that the deposition time have significantly influenced the properties of the prepared thin films. CdS thin film prepared at a deposition time of 20 min showed the optimum characteristics. The photoluminescence measurements showed enhanced structural quality as suggested by the reduction in the intensity of the defect-related emissions. Prolonging the deposition time beyond 20 min does not improve the properties of the grown thin films. Metal-semiconductor-metal photodetectors were fabricated based on the synthesized CdS thin films. Current-voltage measurements showed superior characteristics of the fabricated photodetectors based on CdS thin films grown at deposition times of 10 and 20 min compared to those based on CdS thin film grown at a deposition time of $30 \mathrm{~min}$. Photodetector based on CdS thin film grown at a deposition time of 20 min exhibited enhanced photodetection properties. The device showed the fastest response time of $9 \mathrm{~ms}$ with a photosensitivity of $1440 \%$ to $500 \mathrm{~nm}$ chopped light at an applied bias of $-1 \mathrm{~V}$. Whereas, photodetector based on CdS thin film grown at a deposition time of 30 min showed the longest response and recovery times of 35 and $42 \mathrm{~ms}$, respectively with a photosensitivity of $51.70 \%$. Photodetection measurements revealed that schottky contact based devices have a superior performance than those with ohmic contact-based devices.
\end{abstract}

Keyword: Chemical bath deposition; Deposition time; Nanocrystalline Cds; Photodetectors; Thin films 